\title{
RAD50 deficiency is a predictor of platinum sensitivity in sporadic epithelial ovarian cancers
}

\author{
Adel Alblihy ${ }^{1}$, Muslim L. Alabdullah' ${ }^{1,2}$, Michael S. Toss ${ }^{2}$, Mashael Algethami', Nigel P. Mongan ${ }^{3,4}$, \\ Emad A. Rakha ${ }^{2}$ and Srinivasan Madhusudan ${ }^{1,5,6^{*}}$ (i)
}

\begin{abstract}
Intrinsic or acquired resistance seriously limits the use of platinating agents in advanced epithelial ovarian cancers. Increased DNA repair capacity is a key route to platinum resistance. RAD50 is a critical component of the MRN complex, a 'first responder' to DNA damage and essential for the repair of DSBs and stalled replication forks. We hypothesised a role for RAD50 in ovarian cancer pathogenesis and therapeutics. Clinicopathological significance of RAD50 expression was evaluated in clinical cohorts of ovarian cancer at the protein level $(n=331)$ and at the transcriptomic level $(n=1259)$. Sub-cellular localization of RAD50 at baseline and following cisplatin therapy was tested in platinum resistant (A2780cis, PEO4) and sensitive (A2780, PEO1) ovarian cancer cells. RAD50 was depleted and cisplatin sensitivity was investigated in A2780cis and PEO4 cells. RAD50 deficiency was associated with better progression free survival (PFS) at the protein $(p=0.006)$ and transcriptomic level $(p<0.001)$. Basal level of RAD50 was higher in platinum resistant cells. Following cisplatin treatment, increased nuclear localization of RAD50 was evident in A2780cis and PEO4 compared to A2780 and PEO1 cells. RAD50 depletion using siRNAs in A2780cis and PEO4 cells increased cisplatin cytotoxicity, which was associated with accumulation of DSBs, S-phase cell cycle arrest and increased apoptosis. We provide evidence that RAD50 deficiency is a predictor of platinum sensitivity. RAD50 expression-based stratification and personalization could be viable clinical strategy in ovarian cancers.
\end{abstract}

Keywords: DNA repair, RAD50, Ovarian cancer, Predictive biomarker, Platinum therapy

\section{Introduction}

Platinating agents such as cisplatin and carboplatin are commonly used in the treatment of ovarian cancer. However not all patients respond and the development of intrinsic or acquired resistance to platinum is a formidable clinical problem in ovarian cancers [1]. Platinum compounds form intra-strand and inter-strand adducts, which if not repaired through the nucleotide excision repair (NER) pathway [2,3], can contribute to replication arrest leading to double strand breaks (DSB)

\footnotetext{
* Correspondence: srinivasan.madhusudan@nottingham.ac.uk

${ }^{1}$ Translational Oncology, Division of Cancer \& Stem Cells, School of Medicine, University of Nottingham Biodiscovery Institute, Nottingham NG51PB, UK ${ }^{5}$ Department of Oncology, Nottingham University Hospitals, Nottingham NG51PB, UK

Full list of author information is available at the end of the article
}

accumulation $[4,5]$. DSBs are detected through the DNA damage signalling and response (DDR) mechanisms which coordinate cell cycle response and DNA repair. Increased DNA repair capacity promotes resistance to platinating agents $[6,7]$. On the other hand, reduced DNA repair capacity, such as due to BRCA germ-line deficiency, increase platinum sensitivity. Importantly, PARP inhibitors (Niraparib, Rucaparib, Olaparaib, Talazoparib) are selectively toxic in platinum sensitive BRCA germ line deficient or sporadic epithelial ovarian cancers. Therefore the development of biomarkers that predict platinum sensitivity is an area of unmet clinical need [8].

The MRE11-RAD50-NBS1 (MRN) complex is a 'first responder' to DNA damage and is essential for the repair of DSBs and stalled replication forks [9]. RAD50 is 
a core protein of MRN complex. RAD50 plays a critical role in non-homologous and joining (NHEJ) and homologous recombination (HR) and telomere maintenance $[10,11]$. RAD50 is an ATP-modulated DNA cross-linker that has three vital domains: ATP-binding cassette $(\mathrm{ABC})$, zink hook region and MRE11 interaction site [12], and the integrity of these domains is crucial for the process of DSBs by the MRN complex [13]. RAD50 acts as a bridge at the junction of DNA damage, facilitating the recognition and processing of DNA ends by the exonuclease activity of MRE11 to initiate DNA repair. MRE11 endo- and exo- nuclease activities are stimulated by RAD50 [14]. RAD50 deficiency reduces MRE11 nuclear localization and it is interaction with NBS1. RAD50- deficient cells have impaired cell cycle checkpoints and DNA repair capacity [15]. A role for RAD50 in the maintenance of telomere has been described [16]. Germ-line mutations in RAD50 has been linked to hereditary breast cancers [17]. RAD50 polymorphisms are associated with increased risk of breast and ovarian cancer [18].

We hypothesised a role for RAD50 in ovarian cancer pathogenesis and therapeutics. In the current study we provide evidence that RAD50 deficiency is a predictor of platinum sensitivity in epithelial ovarian cancers.

\section{Results}

RAD50 overexpression is linked to aggressive epithelial ovarian cancers

Clinicopathological significance of RAD50 protein was evaluated by immunohistochemistry in 331 human ovarian cancers (Fig. 1a). Patient demographics are summarized in Supplementary Table 1. RAD50 protein expression was evaluable in 239 tumours. Negative staining, nuclear staining only or nuclear \& cytoplasmic staining was observed in tumours. Intensities of subcellular compartments were each assessed and grouped as follows: $0=$ no staining, $1=$ weak staining, $2=$ moderate

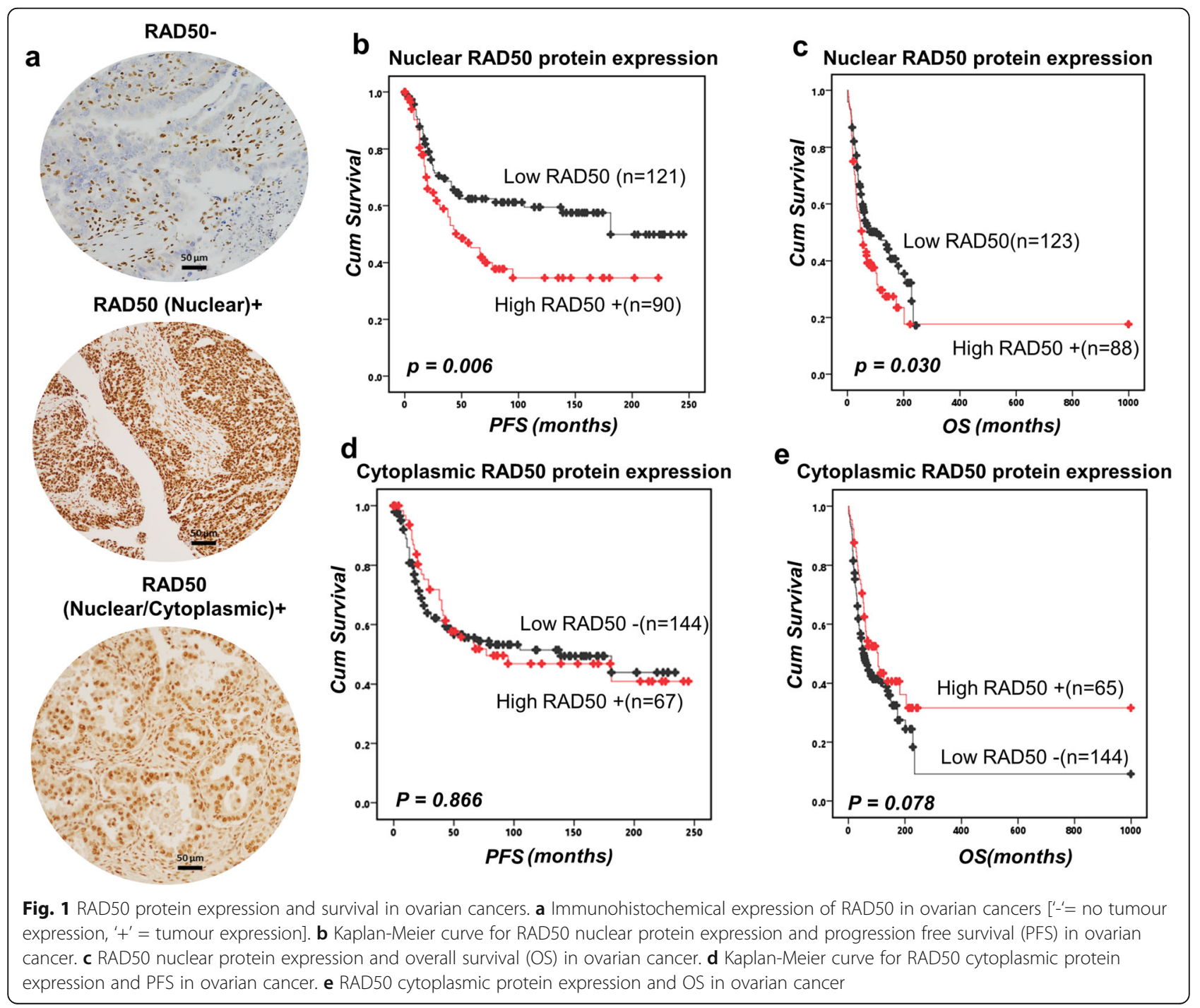


staining, 3 = strong staining. The percentage of tumour cells in each category was estimated (0-100\%). Histochemical score (H-score) (range 0-300) was calculated by multiplying the intensity of staining and the percentage of staining. A median $\mathrm{H}$-score of $\leq 120$ and 0 was used as the cut-off for low RAD50 nuclear and cytoplasmic expression respectively. Full methodology is described in supplementary methods. We correlated nuclear expression and cytoplasmic expression to clinicopathological outcomes and survival. High nuclear RAD50 was seen in 90/239 (42.6\%) tumours and linked to serous cystadenocarcinoma ( $p=$ $0.033)$, high grade 3 tumours $(p=0.004)$ (Supplementary Table 2). Low RAD50 expression was associated with better progression free survival (PFS) $(p=0.006)$ (Fig. 1b) and better overall survival (OS) $(p=0.003)$ (Fig. 1c). Cytoplasmic expression of RAD50 did not influence clinicopathological features or survival (Fig. 1d, e, Supplementary Table 2).

In a multivariate model, high nuclear $\operatorname{RAD} 50(p=0.003)$ was independently associated with PFS. FIGO stage and platinum sensitivity $(p<0.000)$ were also additional factors independently associated with PFS (Table 1). High nuclear $\operatorname{RAD} 50(p=0.011)$, high cytoplasmic $\operatorname{RAD50}(p=0.034)$ along with FIGO stage and platinum sensitivity $(p<$ 0.0001 ) were independent factors associated with OS (Table 1).

For further validation, we investigated RAD50 mRNA expression in a publicly available online gene expression database of 1259 ovarian cancer cases treated with platinum therapy. High mRNA expression of RAD50 significantly associated with poor PFS $(p=0.001)$ (Fig. 2a) but did not influence OS ( $p=0.22)$ (Fig. 2b).

Taken together, the clinical data suggests that RAD50 overexpression could be a predictor of response to platinum therapy. To confirm this hypothesis, we proceeded to pre-clinical studies in platinum sensitive and resistant ovarian cancer cell lines.

\section{RAD50 level at baseline and following cisplatin therapy in ovarian cancer cells}

A2780 cell line is platinum sensitive established from a patient with untreated ovarian cancer. A2780cis cell line is a platinum resistant ovarian cancer developed by continuous exposure of the A2780 cell line to increasing doses of cisplatin. PEO1 platinum sensitive (BRCA2- deficenent) cell line is derived from a patient with a poorly differentiated serous adenocarcinoma treated with platinum-based drugs. PEO4 platinum resistant (BRCA2-proficient) cell line was derived from a malignant effusion from the peritoneal ascites of the same patient after the development of clinical resistance to platinum treatment. The baseline level of RAD50 was investigated in A2780, A2780cis, PEO1 and PEO4 cells. In whole cell lysates, as shown in Fig. 3a, baseline RAD50 protein level was high in A2780cis compared to A2780 cells. Similarly, baseline RAD50 protein level was high in PEO4 compared to PEO1 cells. The quantification of RAD50 baseline levels are shown in Fig. 3b. We then generated nuclear and cytoplasmic extracts at baseline and following $48 \mathrm{~h}$ cisplatin therapy. In platinum resistant A2780cis and PEO4 cells, platinum treatment increased RAD50 nuclear sub-cellular localisation compared to platinum sensitive A2780 and PEO1 cells (Fig. 3c). The quantification of nuclear expression of RAD50 is shown in Fig. 3d. No significant alterations were observed for cytoplasmic expression of RAD50 in A2780, A2780cis, PEO1 and PEO4 cells (Fig. 3e). The data suggests that RAD50 protein expression is subjected to sub-cellular localisation upon cisplatin treatment in A2780cis and PEO4 cells.

\section{RAD50 variant profiling in A2780, A2780cis, PEO1 and PEO4 cells}

Germ-line mutations in RAD50 has been linked to breast and ovarian cancer susceptibility [17]. RAD50 polymorphisms are associated with increased risk of breast and ovarian cancer [18]. We performed targeted deep sequencing for RAD50 variants in A2780, A2780cis, PEO1 and PEO4 cells. Ensembl VEP was used to analyse the effect and location of variants using the HG19/GRCh37 genome version. In the parental A2780 line two unique variants were identified (A: 5:131893147-131893147, a novel variant predicted to alter splicing; B: 5:131977963131977963, rs1804670, a synonymous variant). The Platinum resistant A2780cis harbours a novel unique variant at 5:131973821-131973821 which is predicted to introduce Ala $\rightarrow$ Asp amino acid substitution. This variant is located within the ATPase domain of RAD50. While the $\mathrm{Ala} \rightarrow$ Asp substitution is similar in size and volume, the

Table 1 Multivariate analysis

\begin{tabular}{|c|c|c|c|c|}
\hline \multirow[t]{2}{*}{ Factors } & \multicolumn{2}{|c|}{ Progression free survivals (PFS) } & \multicolumn{2}{|c|}{ Overall survivals (OS) } \\
\hline & $\operatorname{Exp}(95 \% \mathrm{Cl})$ & $P$ value & $\operatorname{Exp}(95 \% \mathrm{Cl})$ & $P$ value \\
\hline RAD50_N & $1.910(1.242,2.938)$ & 0.003 & $1.617(1.117,2.340)$ & 0.011 \\
\hline RAD50_C & $0.874(0.550,1.388)$ & 0.568 & $0.637(0.419,1.077)$ & 0.034 \\
\hline Surgical Pathology Type & $0.969(0.827,1.136)$ & 0.699 & $0.930(0.802,2.418)$ & 0.332 \\
\hline Surgical Pathology Stage & $2.154(1.651,2.812)$ & 0.000 & $1.935(1.549,2418)$ & 0.000 \\
\hline Platinum sensitivity & $20.364(8.642,47.988)$ & 0.000 & $8.412(4.532,15.614)$ & 0.000 \\
\hline
\end{tabular}



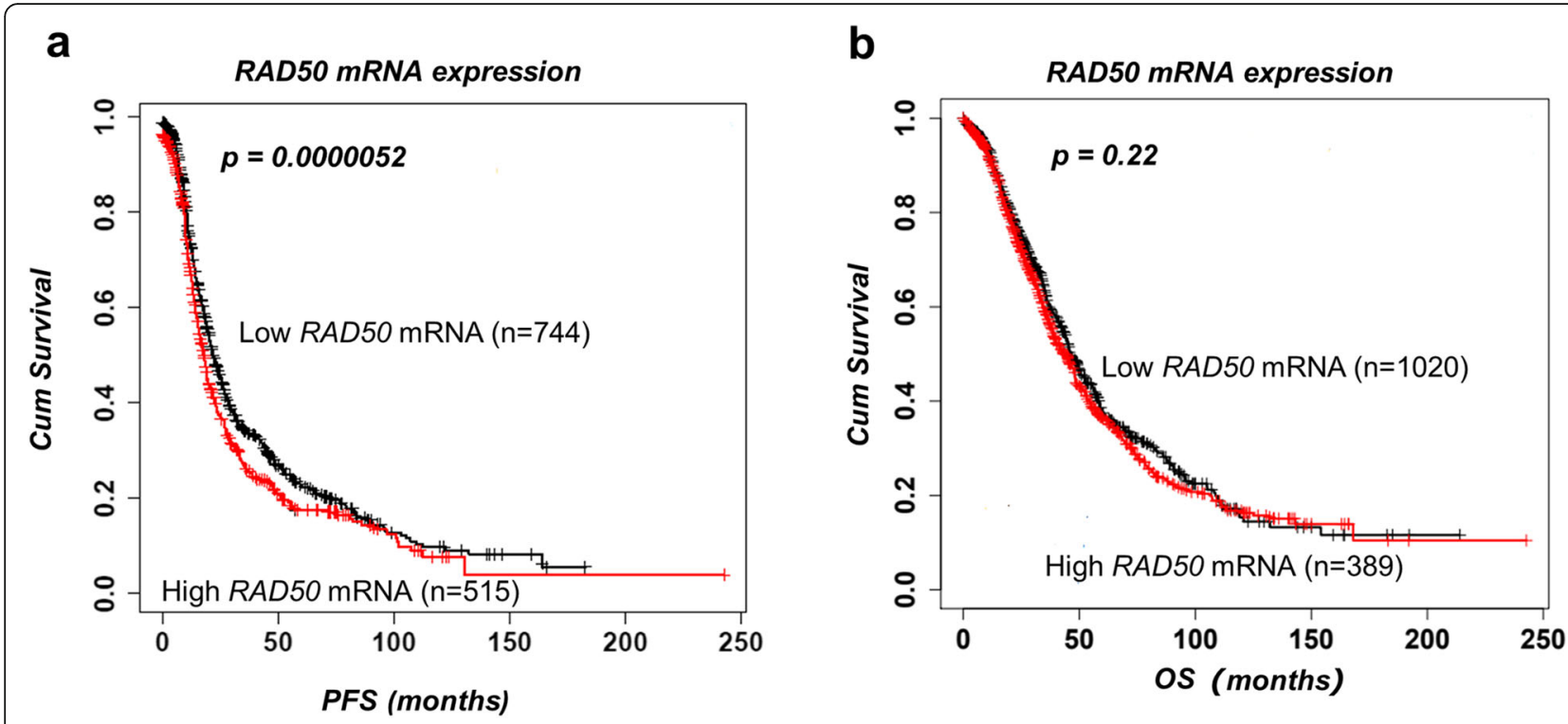

Fig. 2 RAD50 mRNA expression and survival in ovarian cancers. a The association between RAD50 mRNA expression and PFS. b The association between RAD50 mRNA expression and OS

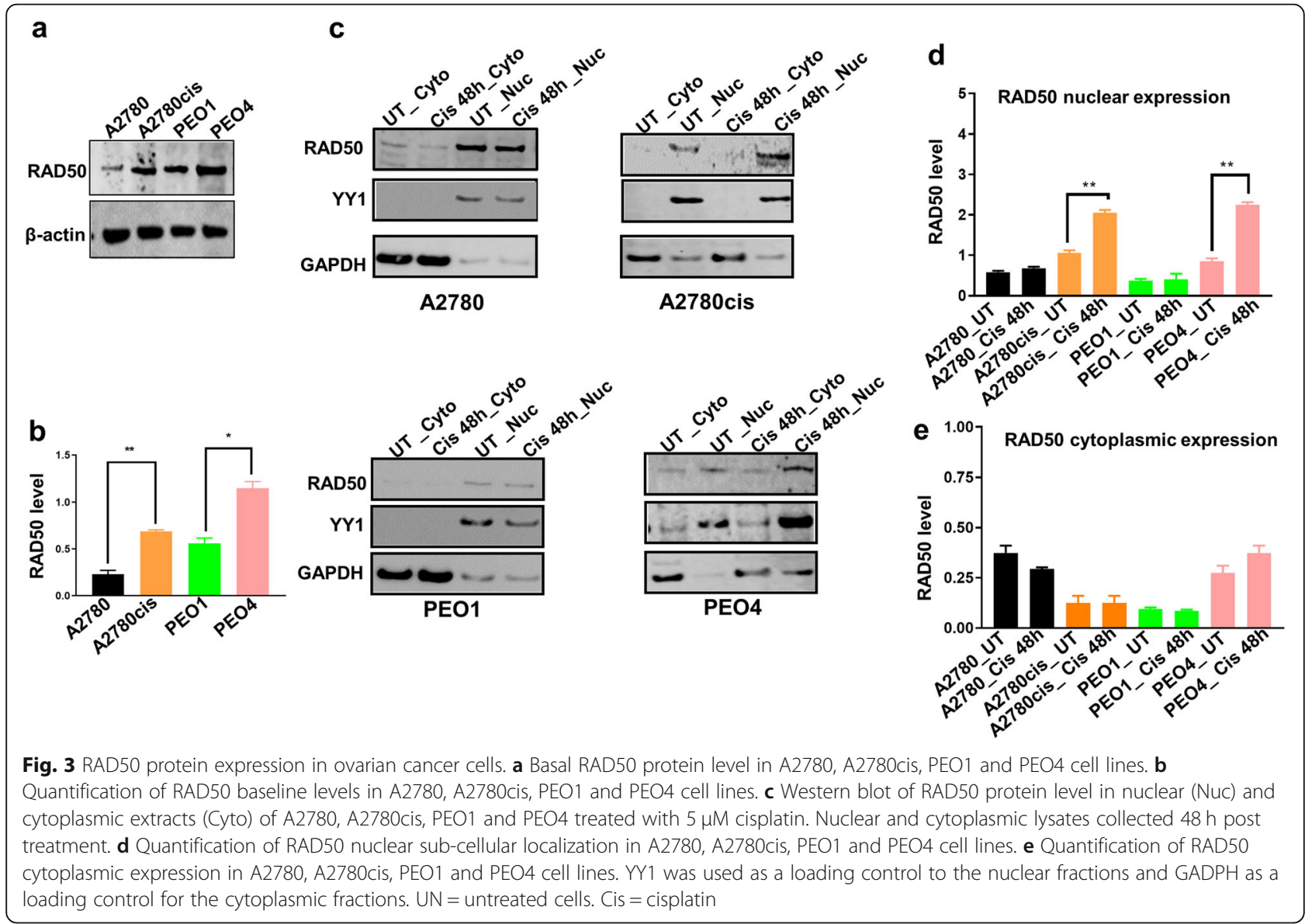


introduction of an acidic aspartic acid may influence substrate access to the ATPase domain. Other mutations in this RAD50 domain are found in colon and stomach cancers [19]. Both PE01 and PE04 both harbour a single known variant (5:131892357-131892357, rs2706335) located upstream of the RAD50 gene which does not alter the coding sequence. We also assessed putative components of the RAD50 interactome (MRE11A, NBN, RINT1, C15ORF26, CELA2B, EP300, GEMIN2, RBBP8, ZFAND2B, ZNF511, RECQL5, MDC1, BARD1, BRCA1, PAXIP1, TERF2, TERF2IP, BLM, DYNLL1, FAM219A, FGFR1OP, H2AFX, ILF2, LRRC39, MAF1, MDM2, PAXIP1, PPARG, TERF2IP, TP53BP1, USP7) for which more than two supporting references were reported in the BioGrid database [20]. Variants were identified in MRE11A, NBN, RINT1, C15ORF26, CELA2B, EP300, GEMIN2, RBBP8, ZFAND2B, ZNF511, RECQL5 in A2780cis and in RECQL5, MDC1 in PE04 (Supplementary Table 3). Taken together, the data provides evidence for a role for variants in RAD50 and its associated complex in platinum resistance in ovarian cancer cell lines.

\section{RAD50 depletion increases cisplatin cytotoxicity in A2780cis and PEO4 cells}

We transiently depleted RAD50 using siRNAs in A2780cis cells (Fig. 4a). The Quantification of western blot showing RAD50 depletion in A2780cis cells is demonstrated in Fig. 4b. As shown in clonogenic assays (Fig. 4c), RAD50_knock down (KD)_A2780cis cells were significantly sensitive to cisplatin treatment compared to scrambled controls. Increased sensitivity was associated with DSB accumulation (Fig. 4d, e), S-phase cell cycle arrest (Fig. 4f, g) and increased apoptosis (Fig. 4h, i) compared to scrambled controls. For further validation, we RAD50 using siRNAs in platinum resistant PEO4 cells (Fig. 5a). The Quantification of western blot showing RAD50 depletion in PEO4 cells is demonstrated in Fig. 5b. As expected, RAD50_KD_PEO4 cells (Fig. 5c)

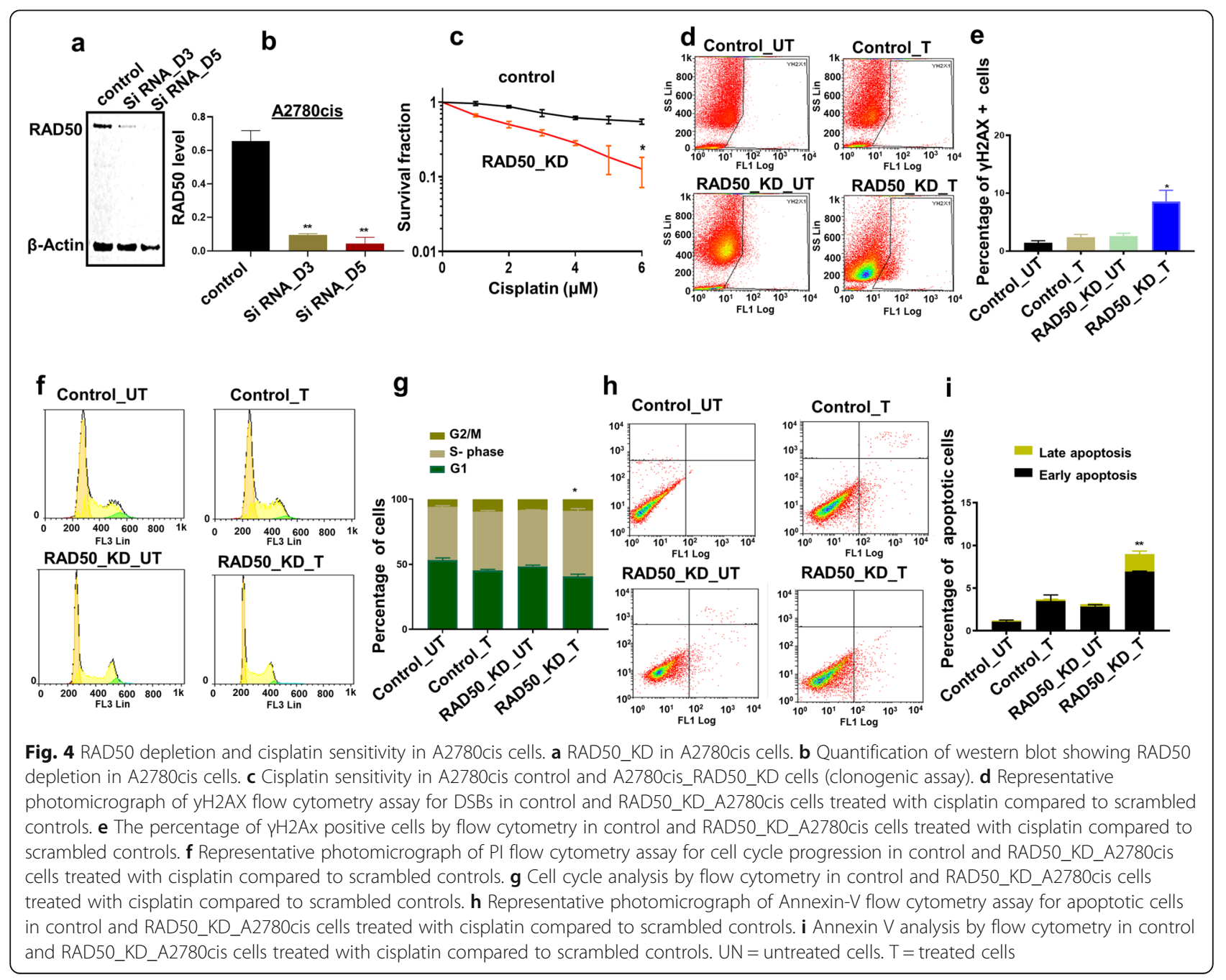




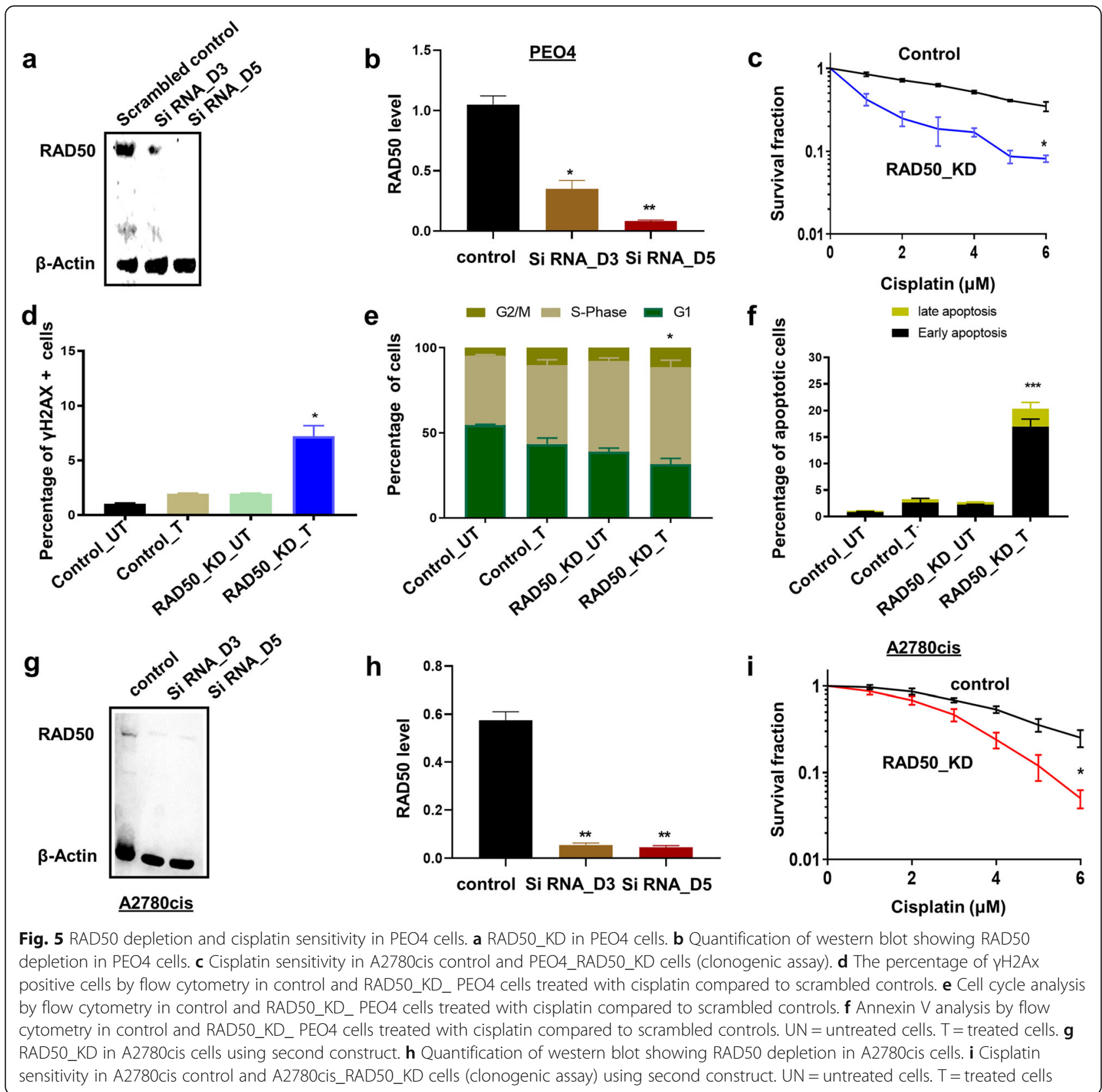

showed increased platinum sensitivity which was associated with DSB accumulation (Fig. 5d), S-Phase arrest (Fig. 5e) and apoptotic cells (Fig. 5f). For additional validation, we tested another siRNA construct for RAD50 depletion in A2780cis cells (Fig.5g). The Quantification of western blot showing RAD50 depletion in A2780cis cells is shown in Fig. 5h. As expected, RAD50 depletion lead to platinum sensitization compared to scrambled controls (Fig. 5i).

Taken together, the clinical and pre-clinical data provides evidence that RAD50 is a predictor of platinum sensitivity in ovarian cancer.

\section{Discussion}

RAD50 has critical roles during HR, NHEJ and telomere maintenance [13]. Here we provide evidence that RAD50 expression is a predictor of platinum sensitivity. High RAD50 expression was associated with aggressive high-grade serous cystadenocarcinomas. In contrast, low RAD50 expression was observed in low-grade epithelial ovarian cancer in another study [21]. At the protein and transcriptomic level, we show that high RAD50 expression was associated with poor PFS in patients. Our data would concur with a previous study in resected nonsmall-cell lung cancer (NSCLC) where high RAD50 
expression was associated with poor survival following radiotherapy [10]. In gastric cancer [22], colorectal cancer [23] and rectal cancers [24], similarly, high RAD50 has been associated with poor clinical outcomes in patients. In the current study cytoplasmic expression of RAD50 was not significantly associated with progression free survival or overall survival. There was a nonsignificant trend of better survival in tumours with high cytoplasmic expression of RAD50. We speculate that cytoplasmic sequestration of DNA repair protein such as RAD50 could result in reduced nuclear levels resulting in improved survival in patients after cisplatin-based chemotherapy. In ovarian cancer cell lines, following cisplatin therapy, we observed increased nuclear accumulation of RAD50 protein in nuclear extracts in platinum resistant cell lines compared to platinum sensitive cell lines. However, a limitation is that we did not validate this observation using confocal microscopy. Nevertheless, RAD50 depletion in platinum resistant ovarian cancer cells increased cisplatin cytotoxicity. Increased toxicity was associated with DSB accumulation as evidenced In a head and neck squamous cell carcinoma model, RAD50 blockade resulted in cisplatin chemosensitization [13]. Furthermore, RAD50 depletion also sensitized human breast cancer cells to cisplatin treatment [18]. Disrupting RAD50 function has been shown to sensitize human nasopharyngeal carcinoma cells to radiotherapy [12]. In NSCLC cells, RAD50 depletion not only increased radiation-sensitivity but RAD50 overexpression enhanced radio-resistance in vitro [10]. The data would suggest that RAD50 is a predictor of response to DNA damaging cytotoxic therapy.

Germ-line mutations in RAD50 has been linked to breast and ovarian cancer susceptibility [17]. RAD50 polymorphisms are also associated with increased risk of breast and ovarian cancer [18]. In ovarian cancer cell lines, however, RAD50 variants have not been described previously. Therefore, we conducted a deep sequencing study and identified a unique RAD50 variant at 5:131973821-131973821 in A2780cis cells which is predicted to alter RAD50 function. However, detailed mechanistic study would be required to evaluate whether the variation contributes to platinum resistance. In addition, as RAD50 is multi-functional protein with previously reported interacting partners, we conducted a bioinformatic analyses to understand RAD50 interactome in ovarian cancer cells. The interactome revealed several partners (including key DNA repair genes such as NBS1, Mre11) with roles in platinum resistance. Together, the data provides evidence that RAD50 directly and indirectly (through its interactors) influence response to platinum induced DNA damage in cells.
BRCA1 interacts with RAD50 [25, 26] during DSB repair. In addition, during early stages of DSB repair via HR, DSBs activate ATM and ATR kinases which in turn phosphorylate p53 and BRCA1. During subsequent stages of HR, MRN resects the DNA to form 3" overhangs. This is followed by loading of RAD51 onto RPAcoated DNA under the influence of BRCA2 [26]. BRCA1 germ-line deficiency or RAD50 inactivation results in defective HR. Interestingly, a link between RAD50 deficiency, BRCAness phenotype and PARP inhibitor sensitivity has been shown in ovarian cancers [27]. In $B R C A$ wild-type ovarian cancers, $R A D 50$ deletion was shown in $18 \%$ of tumours and correlated better PFS and OS. RAD50 depletion in ovarian cancer cell lines also increased response to PARP inhibitor therapy [27]. The data including ours provide evidence that RAD50 deficiency is not only a marker of platinum sensitivity but could also predict response to PARP inhibitor therapy in epithelial ovarian cancers.

\section{Materials and methods \\ Clinical study \\ RAD50 expression level in ovarian cancers}

Evaluation of the expression of RAD50 was performed on tissue microarrays of 331 consecutive sporadic epithelial ovarian cancer cases treated at Nottingham University Hospitals (NUH) between 1997 and 2010. This study was carried out in accordance with the declaration of The Helsinki. The study was approved by the Nottingham Research Biorepository (NRB) Access Committee under the biobank ethical approval REC reference: 10/H1008/72 (NRES Committee North West - Greater Manchester Central). All patients provided informed consent. Patient demographics is summarized in Supplementary Table 1. Detailed methodology for immunohistochemical evaluation of RAD50 expression and statistical analyses is described in supplementary methods.

\section{RAD50 transcript in ovarian cancers}

RAD50 mRNA expression was evaluated in a publically available online gene expression dataset of 1259 ovarian cancer patients treated with platinum-based chemotherapy from 15 previously published studies and available at "http:// kmplot.com/analysis/index.php? $\mathrm{p}=$ service\&cancer=ovar'.

\section{Pre-clinical study \\ Cell lines and tissue culture}

A2780 (platinum sensitive) A2780cis (platinum resistant) were purchased from Sigma Aldrich (Gillingham, UK). PEO1 (BRCA2-deficient, platinum sensitive) and PE04 (BRCA2-proficient, platinum resistant) were purchased from American Type Culture Collection (ATCC, Manassas, USA). Cells cultured in RPMI (R8758, Merck, UK) supplemented with 10\% FBS (F4135, Merck, UK), 1\% 
Penicillin-Streptomycin (P4333, Merck, UK). All cell lines were maintained in a humidified incubator at $37^{\circ} \mathrm{C}$ in a $5 \% \mathrm{CO} 2$ atmosphere.

\section{Western blot analysis}

Cells were trypsinized and lysed in RIPA buffer (R0278, Sigma.UK) with the addition of protease cocktail inhibitor (P8348, Sigma, UK), phosphatase inhibitor cocktail 2 (P5726, Sigma, UK) and phosphatase inhibitor cocktail 3 (P0044, Sigma) and stored at $-20^{\circ} \mathrm{C}$. Protein quantification was performed using BCA Protein Assay kit (23225, Thermofisher, UK). Samples were run on SDS-bolt gel (4-12\%) bis-tris. Membranes were then incubated with primary antibodies as follows: RAD50 (1:500, ab89), Bactin (1:1000, ab8226), YY1 (1:1000, ab109228), GADPH $(1: 1000, a b 9485)$. Membranes were then washed and incubated with Infrared dye-labelled secondary antibodies (LiCor) [IRDye 800CW Donkey Anti-Rabbit IgG (92632213) and IRDye 680CW Donkey Anti-Mouse IgG (926-68072)] at dilution of 1:10,000 for $1 \mathrm{~h}$. Membranes were scanned with a LiCor Odyssey machine (700 and $800 \mathrm{~nm}$ ) to determine protein levels.

\section{Nuclear and cytoplasmic extracts}

Cells were seeded in T25 flasks overnight. Cells were then treated with $5 \mu \mathrm{M}$ of cisplatin and left at $37^{\circ} \mathrm{C}$ in a $5 \% \mathrm{CO} 2$ atmosphere for $48 \mathrm{~h}$. Cells were then harvest by trypsinization, washed with PBS and centrifuged at $1000 \times \mathrm{g}$ for $5 \mathrm{~min}$. Cell Lysates were extracted using the NE-PER Nuclear and Cytoplasmic Extraction Reagents (78833, Thermofisher, UK). Cells were collected by trypsinization, washed with PBS and centrifuged at $1000 \times \mathrm{g}$ for $5 \mathrm{~min}$. Extracts were quantified using BCA protein quantification kit (Thermo Fisher Scientific) and protein levels were checked by western blot. YY1 was used as a loading control to the nuclear fractions and GADPH as a loading control for the cytoplasmic fractions.

\section{Transient knockdowns of RAD50}

RAD50 (ID S792) and the validation construct of RAD50 (ID S793) siRNAs oligonucleotides were purchased from Invitrogen. Lipofectamine 3000 reagent (L3000015, Invitrogen, UK) was used according to the manufacturer's protocol. Briefly cells were seeded at 50 $60 \%$ confluency in T25 flasks overnight. Cells were transfected with $20 \mathrm{nM}$ of siRNA oligonuclotide or scrambled SiRNA oligonucleotide control (4390843, Thermofiher) in Opti-MEM media (31985-062, Gibco).

\section{Clonogenic assays}

In the clonogenic assay, 32 cells $/ \mathrm{cm}^{2}$ were seeded in 6well plates and left at $37^{\circ} \mathrm{C}$ in a $5 \% \mathrm{CO}_{2}$ atmosphere. Cisplatin (Kindly provided by Nottingham University Hospital) was added at the indicated concentrations and the plates were left at $37^{\circ} \mathrm{C}$ in a $5 \% \mathrm{CO} 2$ atmosphere for 14 days. Later the plates were washed with PBS, fixed and stained and colonies were counted. Survival fraction (SF) were calculated using the formula SF $=$ no. of colonies formed after treatment/no. of cells seeded x platting efficiency. Number of colonies counted were normalised relative to the count of untreated wells which were considered as $100 \%$ survival.

\section{Cell cycle and apoptosis by flow Cytometry}

$1 \times 10^{5}$ Cells per well were seeded in 6-well plates overnight. Cells were treated with Cisplatin $(1 \mu \mathrm{M})$ for A2780 cells and $(5 \mu \mathrm{M})$ for A2780 cis cells. After $24 \mathrm{~h}$ Cells were trypsinized and washed with ice cold PBS, then fixed in $70 \%$ ethanol for at least 30 mins. After removal of the fixative solution by centrifugation cells were stained with phospho Histone $(\gamma \mathrm{H} 2 \mathrm{AX})$ Ser139. Cells were then treated with RNase and DNA content were stained with $10 \mu \mathrm{g} / \mathrm{ml}$ propidium iodide (Sigma Aldrich) in PBS. For Apoptosis detection, cells were collected by trypsinization after $24 \mathrm{~h}$ washed and analysed using annexinV detection kit (BD biosciences). Samples were analysed on FC500 flow cytometer (Beckman Coulter) and data were analysed using Weasel software. Data were generated using GraphPad Prism7 software.

\section{Statistical analysis}

Data was conducted as on GraphPad Prism 7 software. To compare between two groups, student- Ttests analysis was performed. One-way ANOVA was performed to compare between more than two groups (variances analyses). Two-way ANOVA was used to analyse two variables such as Annexin $\mathrm{V}$ analysis and cell cycle analysis. All experiments were expressed as means \pm standard deviation S.D. of three independent experiments. $p$-values $<0.05=*$, $p$-value $<0.01=* * \&$ $p$-value $<0.001=* * *$.

\section{Targeted next generation sequencing and bioinformatics}

Genomic DNA was extracted from cell lines using the PicoPure $^{\text {th }}$ DNA Extraction Kit (Thermofisher,UK). Targeted next generation sequencing was used to identify genomic variants in platinum sensitive (A2780) and platinum resistant derivatives (A2780cis). The SureSelect All Exon V5 kit (Agilent Technologies) was used to enrich for protein coding regions and sequencing performed using an Illumina NextSeq500 sequencer with paired end reads $(150 \mathrm{bp})$ and a minimum of 88 million reads generated per sample. Raw reads were fastq formatted. Contaminating adapter sequences and low-quality sequences were processed using Skewer [28]. Quality processed reads were aligned to the HG19 reference genome using BWA [29], duplicate alignments identified and processed using PicardTools, and realignment completed 
using the Abra assembly based realigner [30] to enhance detection of insertion/deletion variants. Variant calling and filtering was completed using Samtools/Bcftools (v1.3.1) [31]. Variants, in variant call format (VCF), associated with Platinum resistance were identified using Vcftools [32]. Variants were annotated and functional significance assessed using the Ensembl Variant Effect Predictor tool [33]. Library preparation and sequencing was conducted by Source Biosciences (Nottingham, UK).

In accordance with the journal's guidelines, we will provide our data for the reproducibility of this study in other centres if such is requested.

\section{Supplementary Information}

The online version contains supplementary material available at https://doi. org/10.1186/s43556-020-00023-y.

Additional file 1: Supplementary Table 1. Patient demographics and pathological features in ovarian cancer. Supplementary Table 2. The correlation between RAD50 nuclear and cytoplasmic expression and clinicopathological parameters. Supplementary Table 3. Cell cycle quantification following cisplatin treatment in control and RAD50_KD cell lines. Supplementary methods.

\section{Abbreviations}

ABC: ATP-binding cassette; DDR: DNA damage signalling and response; DSB: Double strand break; HR: Homologues recombination; MRN: The MRE11-RAD50-NBS1; NER: Nucleotide excision repair; NHEJ: Non-homologous and joining; OS: Overall survivals; PFS: Progression free survivals

\section{Acknowledgements}

None

\section{Consent to participate}

All patients provided informed consent.

\section{Authors' contributions}

A.A: Data curation (IHC, Tissue culture studies); Formal analysis; Investigation; Methodology; Resources; Roles/Writing - original draft; Writing - review \& editing. M.L.A: Data curation (IHC); Formal analysis; Investigation; Methodology; Resources; Roles/Writing - original draft; Writing - review \& editing. M.S.T: Data curation (IHC); Formal analysis; Investigation; Methodology; Resources; Roles/Writing - original draft; Writing - review \& editing. M.A: Data curation (Tissue culture); Formal analysis; Investigation; Methodology; Resources; Roles/Writing - original draft; Writing - review \& editing. N.P.M: Data curation (bioinformatics); Formal analysis; Investigation; Methodology; Resources; Roles/Writing - original draft; Writing - review \& editing. E.A.R: Data curation (IHC); Formal analysis; Investigation; Methodology; Resources; Roles/Writing - original draft; Writing - review \& editing. S.M: Data curation (IHC, Tissue culture studies); Formal analysis; Investigation; Methodology; Resources; Roles/Writing - original draft; Writing - review \& editing. The author(s) read and approved the final manuscript.

\section{Funding}

This Research work was supported by Naaz-Coker Ovarian Cancer Fellowship (University of Nottingham, UK)

\section{Availability of data and materials}

All data and material relevant to this publication is available upon reasonable request.

\section{Ethics approval and consent to participate}

The study was approved by the Nottingham Research Biorepository (NRB) Access Committee under the biobank ethical approval REC reference: 10/ H1008/72 (NRES Committee North West - Greater Manchester Central).

\section{Consent for publication}

Yes.

\section{Competing interests}

The authors disclose no potential conflicts of interest.

\section{Author details}

${ }^{1}$ Translational Oncology, Division of Cancer \& Stem Cells, School of Medicine, University of Nottingham Biodiscovery Institute, Nottingham NG51PB, UK. ${ }^{2}$ Academic Pathology, Division of Cancer \& Stem Cells, School of Medicine, University of Nottingham Biodiscovery Institute, Nottingham NG51PB, UK.

${ }^{3}$ Faculty of medicine and Health Sciences, Centre for Cancer Sciences, University of Nottingham, Sutton Bonington Campus, Sutton Bonington, Leicestershire LE12 5RD, UK. ${ }^{4}$ Department of Pharmacology, Weill Cornell Medicine, New York, NY 10065, USA. ${ }^{5}$ Department of Oncology, Nottingham University Hospitals, Nottingham NG51PB, UK. ${ }^{6}$ Division of Cancer \& Stem Cells, School of Medicine, Nottingham Biodiscovery Institute, University of Nottingham, University Park, Nottingham NG7 3RD, UK.

Received: 26 November 2020 Accepted: 30 November 2020 Published online: 30 December 2020

\section{References}

1. Mesquita KA, Alabdullah M, Griffin M, Toss MS, Fatah T, Alblihy A, et al, ERCC1-XPF deficiency is a predictor of olaparib induced synthetic lethality and platinum sensitivity in epithelial ovarian cancers. Gynecol Oncol. 2019; 153(2):416-24. https://doi.org/10.1016/j.ygyno.2019.02.014.

2. Spivak G. Nucleotide excision repair in humans. DNA Repair (Amst). 2015;36: 13-8. https://doi.org/10.1016/j.dnarep.2015.09.003.

3. Scharer OD. Nucleotide excision repair in eukaryotes. Cold Spring Harb Perspect Biol. 2013;5(10):a012609. https://doi.org/10.1101/cshperspect. a012609.

4. Lopez-Martinez D, Liang C-C, Cohn MA. Cellular response to DNA interstrand crosslinks: the Fanconi anemia pathway. Cell Mol Life Sci. 2016; 73(16):3097-114. https://doi.org/10.1007/s00018-016-2218-x.

5. Basourakos SP, Li L, Aparicio AM, Corn PG, Kim J, Thompson TC. Combination platinum-based and DNA damage response-targeting cancer therapy: evolution and future directions. Curr Med Chem. 2017;24(15):1586606. https://doi.org/10.2174/0929867323666161214114948.

6. Rabik CA, Dolan ME. Molecular mechanisms of resistance and toxicity associated with platinating agents. Cancer Treat Rev. 2007;33(1):9-23. https://doi.org/10.1016/j.ctrv.2006.09.006.

7. Kartalou M, Essigmann JM. Mechanisms of resistance to cisplatin. Mutat Res. 2001;478(1-2):23-43. https://doi.org/10.1016/s0027-5107(01)00141-5.

8. Ali R, Alabdullah M, Alblihy A, Miligy I, Mesquita KA, Chan SY, et al. PARP1 blockade is synthetically lethal in XRCC1 deficient sporadic epithelial ovarian cancers. Cancer Lett. 2020;469:124-33. https://doi.org/10.1016/j.canlet.2019. 10.035 .

9. Syed A, Tainer JA. The MRE11-RAD50-NBS1 complex conducts the orchestration of damage signaling and outcomes to stress in DNA replication and repair. Annu Rev Biochem. 2018;87:263-94. https://doi.org/ 10.1146/annurev-biochem-062917-012415.

10. Wang Y, Gudikote J, Giri U, Yan J, Deng W, Ye R, et al. RAD50 expression is associated with poor clinical outcomes after radiotherapy for resected nonsmall cell lung cancer. Clin Cancer Res. 2018;24(2):341-50. https://doi.org/10. 1158/1078-0432.CCR-17-1455.

11. Zhu XD, Kuster B, Mann M, Petrini JH, de Lange T. Cell-cycle-regulated association of RAD50/MRE11/NBS1 with TRF2 and human telomeres. Nat Genet. 2000;25(3):347-52. https://doi.org/10.1038/77139.

12. Chang L, Huang J, Wang K, Li J, Yan R, Zhu L, et al. Targeting Rad50 sensitizes human nasopharyngeal carcinoma cells to radiotherapy. BMC Cancer. 2016;16(1):190. https://doi.org/10.1186/s12885-016-2190-8.

13. Abuzeid WM, Jiang X, Shi G, Wang H, Paulson D, Araki K, et al. Molecular disruption of RAD50 sensitizes human tumor cells to cisplatin-based chemotherapy. J Clin Invest. 2009;119(7):1974-85. https://doi.org/10.1172/ JCl33816.

14. Waltes R, Kalb R, Gatei M, Kijas AW, Stumm M, Sobeck A, et al. Human RAD50 deficiency in a Nijmegen breakage syndrome-like disorder. Am J Hum Genet. 2009;84(5):605-16. https://doi.org/10.1016/j.ajhg.2009.04.010.

15. Gatei M, Jakob B, Chen P, Kijas AW, Becherel OJ, Gueven N, et al. ATM protein-dependent phosphorylation of Rad50 protein regulates DNA repair 
and cell cycle control. J Biol Chem. 2011;286(36):31542-56. https://doi.org/ 10.1074/jbc.M111.258152.

16. Chai W, Sfeir AJ, Hoshiyama H, Shay JW, Wright WE. The involvement of the Mre11/Rad50/Nbs1 complex in the generation of G-overhangs at human telomeres. EMBO Rep. 2006;7(2):225-30. https://doi.org/10.1038/sj.embor. 7400600.

17. Heikkinen K, Rapakko K, Karppinen SM, Erkko H, Knuutila S, Lundán T, et al. RAD50 and NBS1 are breast cancer susceptibility genes associated with genomic instability. Carcinogenesis. 2006;27(8):1593-9. https://doi.org/10. 1093/carcin/bgi360.

18. Flores-Perez A, Rafaelli LE, Ramirez-Torres N, Arechaga-Ocampo E, Frias S, Sanchez S, et al. RAD50 targeting impairs DNA damage response and sensitizes human breast cancer cells to cisplatin therapy. Cancer Biol Ther. 2014;15(6):777-88. https://doi.org/10.4161/cbt.28551.

19. Chen H, Li C, Peng X, Zhou Z, Weinstein JN, Cancer Genome Atlas Research $\mathrm{N}$, et al. A pan-cancer analysis of enhancer expression in nearly 9000 patient samples. Cell. 2018;173(2):386-399 e312. https://doi.org/10.1016/j.cell.2018. 03.027.

20. Oughtred R, Stark C, Breitkreutz BJ, Rust J, Boucher L, Chang C, et al. The BioGRID interaction database: 2019 update. Nucleic Acids Res. 2019;47(D1): D529-41. https://doi.org/10.1093/nar/gky1079.

21. Brandt S, Samartzis EP, Zimmermann AK, Fink D, Moch H, Noske A, et al. Lack of MRE11-RAD50-NBS1 (MRN) complex detection occurs frequently in low-grade epithelial ovarian cancer. BMC Cancer. 2017;17(1):44. https://doi. org/10.1186/s1 2885-016-3026-2.

22. Altan B, Yokobori T, Ide M, Bai T, Yanoma T, Kimura A, et al. High expression of MRE11-RAD50-NBS1 is associated with poor prognosis and chemoresistance in gastric cancer. Anticancer Res. 2016;36(10):5237-47. https://doi.org/10.21873/anticanres.11094.

23. Chen C, Wang Y, Mei JF, Li SS, Xu HX, Xiong HP, et al. Targeting RAD50 increases sensitivity to radiotherapy in colorectal cancer cells. Neoplasma. 2018;65(1):75-80. https://doi.org/10.4149/neo_2018_170219N128.

24. Ho V, Chung L, Singh A, Lea V, Abubakar A, Lim SH, et al. Overexpression of the MRE11-RAD50-NBS1 (MRN) complex in rectal cancer correlates with poor response to neoadjuvant radiotherapy and prognosis. BMC Cancer 2018;18(1):869. https://doi.org/10.1186/s1 2885-018-4776-9.

25. Zhong Q, Chen CF, Li S, Chen Y, Wang CC, Xiao J, et al. Association of BRCA1 with the hRad50-hMre11-p95 complex and the DNA damage response. Science. 1999;285(5428):747-50. https://doi.org/10.1126/science. 285.5428.747.

26. Roy R, Chun J, Powell SN. BRCA1 and BRCA2: different roles in a common pathway of genome protection. Nat Rev Cancer. 2011;12(1):68-78. https:// doi.org/10.1038/nrc3181.

27. Zhang M, Liu G, Xue F, Edwards R, Sood AK, Zhang W, et al. Copy number deletion of RAD50 as predictive marker of BRCAness and PARP inhibitor response in BRCA wild type ovarian cancer. Gynecol Oncol. 2016;141(1):5764. https://doi.org/10.1016/j.ygyno.2016.01.004.

28. Jiang H, Lei R, Ding SW, Zhu S. Skewer: a fast and accurate adapter trimmer for next-generation sequencing paired-end reads. BMC Bioinformatics. 2014; 15:182. https://doi.org/10.1186/1471-2105-15-182

29. Li H, Durbin R. Fast and accurate long-read alignment with burrowswheeler transform. Bioinformatics. 2010;26(5):589-95. https://doi.org/10. 1093/bioinformatics/btp698.

30. Mose LE, Wilkerson MD, Hayes DN, Perou CM, Parker JS. ABRA: improved coding indel detection via assembly-based realignment. Bioinformatics. 2014;30(19):2813-5. https://doi.org/10.1093/bioinformatics/btu376.

31. Li H, Handsaker B, Wysoker A, Fennell T, Ruan J, Homer N, et al. The sequence alignment/map format and SAMtools. Bioinformatics. 2009;25(16): 2078-9. https://doi.org/10.1093/bioinformatics/btp352.

32. Danecek P, Auton A, Abecasis G, Albers CA, Banks E, DePristo MA, et al. The variant call format and VCFtools. Bioinformatics. 2011;27(15):2156-8. https:// doi.org/10.1093/bioinformatics/btr330.

33. McLaren W, Gil L, Hunt SE, Riat HS, Ritchie GR, Thormann A, et al. The Ensembl variant effect predictor. Genome Biol. 2016;17(1):122. https://doi. org/10.1186/s13059-016-0974-4

\section{Publisher's Note}

Springer Nature remains neutral with regard to jurisdictional claims in published maps and institutional affiliations. 